\title{
POTENTIAL OF SEVERAL ARBITRARILY LOCATED DISKS
}

\author{
V. I. FABRIKANT ${ }^{1}$
}

(Received 8 April 1986; revised 2 November 1986)

\begin{abstract}
The electrostatic field of a set of arbitrarily located circular disks is considered. A set of governing integral equations is derived by a new method. It is shown that some integral characteristics can be found without solving the integral equations. The upper and lower bounds for the total charge are found from a set of linear algebraic equations whose coefficients are defined by simple geometric characteristics of the system. Examples considered show sufficient sharpness of the estimations.
\end{abstract}

\section{Introduction}

The problem of charged coaxial disks has been attracting the attention of scientists since the last century. Kirchhoff, Ignatowsky, Nomura, Cooke and others made a significant contribution to its solution. A comprehensive literature review can be found in $[4,5]$. There are just a few papers where the problem of two coplanar disks is considered; among them we know of only one [2] where some numerical results of sufficient accuracy are given. We are aware of only one publication [3] treating the problem of two non-parallel disks, whose "centres" are located in one plane orthogonal to the planes of both disks, by the Mehler-Fok transform, with consequent use of the small-parameter method. To the best of our knowledge there are no publications considering the electrostatic problem of two or more arbitrarily located disks, mainly due to the fact that existing methods are not capable of solving these problems.

Here, a new approach is suggested providing a direct and elementary derivation of the set of governing integral equations, which can be solved by iteration.

\footnotetext{
${ }^{1}$ Department of Mechanical Engineering, Concordia University, Montreal, H3G 1M8, Canada (C) Copyright Australian Mathematical Society 1988, Serial-fee code 0334-2700/88
} 
One can obtain upper and lower bounds for certain integral characteristics without solving the integral equations. For example, the value of the total charge can be assessed by solving a set of linear algebraic equations. Two examples are considered; the first one, involving two disks, in order to compare our results with those available in the literature, and to establish that the discrepancy between our central assessment and the total charge evaluated in [2] is less than $0.4 \%$. A new type of spatial configuration of $n+1$ disks is considered in the second example.

\section{Formulation of the problem and its solution.}

Consider a system of $n$ charged arbitrarily-located circular disks. Let $a_{i}$ be the radius of the $i$ th disk, and $S_{i}$ be its surface. We can single out, without loss of generality, disk number one and place the origin of the set of cylindrical coordinates $(\rho, \phi, z)$ at its centre so that the $O z$ axis is orthogonal to the disk's plane. Let the position vector $\mathbf{r}_{i}$ indicate the centre of the $i$ th disk, and the unit vector $\mathbf{n}_{\mathbf{z}}$, orthogonal to the disk's plane, indicate its orientation. The problem is to find the electrostatic potential due to the system of charged disks; that is, to find a harmonic function $V(\rho, \phi, z)$, satisfying the following boundary conditions:

$$
V(\rho, \phi, z)=v_{i}(\rho, \phi, z) \quad \text { for }(\rho, \phi, z) \subset S_{i} ; i=1,2, \ldots, n \text {. }
$$

The potential can be represented by a simple layer distribution as follows:

$$
V=\sum_{i=1}^{n} \iint_{S_{i}} \frac{q_{i}}{R_{i}} d S_{i}
$$

Here, $q_{i}$ are the (as yet unknown) charge densities, and $R_{i}$ stands for the distance between a point of integration inside $S_{i}$ and an arbitrary point in the space.

Now make use of the following integral representation for the reciprocal distance [1]:

$$
\frac{1}{\left[\rho^{2}+\rho_{i}^{2}-2 \rho \rho_{i} \cos \left(\phi-\phi_{1}\right)+z_{i}^{2}\right]^{1 / 2}}=\frac{2}{\pi} \int_{0}^{c_{i}(\rho)} \frac{\lambda\left(\frac{x^{2}}{\rho \rho_{i}}, \phi-\phi_{i}\right) d x}{\left[\left(\rho_{i}^{2}-x^{2}\right)\left(\rho^{2}-g_{i}^{2}(x)\right)\right]^{1 / 2}}
$$

where

$$
\begin{gathered}
g_{\imath}^{2}(x)=x^{2}\left[1+\frac{z_{i}^{2}}{\rho_{i}^{2}-x^{2}}\right] \\
\lambda(k, \psi)=\frac{1-k^{2}}{1+k^{2}-2 k \cos \psi}
\end{gathered}
$$

and

$$
c_{i}(\rho)=\frac{1}{2}\left\{\left[\left(\rho+\rho_{i}\right)^{2}+z_{i}^{2}\right]^{1 / 2}-\left[\left(\rho-\rho_{i}\right)^{2}+z_{i}^{2}\right]^{1 / 2}\right\} .
$$


An obvious simplification of (3) is valid when $z_{i}=0$, namely

$$
\frac{1}{\left[\rho^{2}+\rho_{i}^{2}-2 \rho \rho_{i} \cos \left(\phi-\phi_{i}\right)\right]^{1 / 2}}=\frac{2}{\pi} \int_{0}^{\min \left(\rho, \rho_{i}\right)} \frac{\lambda\left(\frac{x^{2}}{\rho \rho_{i}}, \phi-\phi_{i}\right) d x}{\left[\left(\rho_{i}^{2}-x^{2}\right)\left(\rho^{2}-x^{2}\right)^{1 / 2}\right.} .
$$

Substituting the boundary conditions (1) for the first disk into (2) and using (3) and (7) yields the following integral equation:

$$
\begin{aligned}
4 \int_{0}^{\rho} & \frac{a x}{\sqrt{ }\left(\rho^{2}-x^{2}\right)} \int_{x}^{\tilde{n}_{i}} \frac{r d r}{\sqrt{ }\left(r^{2}-x^{2}\right)} L\left(\frac{x^{2}}{\rho r}\right) q_{1}(r, \phi) \\
& +\frac{2}{\pi} \sum_{i=2}^{n} \iint_{S_{i}}\left[\int_{0}^{c_{i}(\rho)} \frac{\lambda\left(\frac{x^{2}}{\rho \rho_{i}}, \phi-\phi_{i}\right) d x}{\left[\left(\rho_{i}^{2}-x^{2}\right)\left(\rho^{2}-g_{i}^{2}(x)\right)\right]^{1 / 2}}\right] q_{i} d S_{i}=v_{1}(\rho, \phi),
\end{aligned}
$$

where the Poisson type $L$-operator is introduced as

$$
L(k) f(\phi)=\frac{1}{2 \pi} \int_{0}^{2 \pi} \lambda(k, \phi-\psi) f(\psi) d \psi
$$

The following well-known properties of the $L$-operators will be used here

$$
L(k) L\left(k_{1}\right)=L\left(k k_{1}\right) \quad \text { for } k<1, k_{1}<1 ; \quad \lim _{k \rightarrow 1-} L(k) f=f .
$$

Let us apply the operator

$$
L\left(\frac{\eta}{y}\right) \frac{d}{d y} \int_{0}^{y} \frac{\rho d \rho}{\sqrt{ }\left(y^{2}-\rho^{2}\right)} L(\rho / \eta)
$$

to both sides of (8). Here we have introduced a dummy parameter $\eta$, in order to keep the parameter of the $L$-operator dimensionless and less than unity. We call the parameter $\eta$ a 'dummy' because it was introduced for some formal reasons only; it will disappear in the final result, and therefore has no bearing on the procedure to follow. The result of application of the operator above is

$$
\begin{aligned}
& 2 \pi \int_{y}^{a_{1}} \frac{r d r}{\sqrt{ }\left(r^{2}-y^{2}\right)} L\left(\frac{y}{r}\right) q_{1}(r, \phi) \\
& \quad+\sum_{i=2}^{n} \iint_{S_{i}} \frac{\lambda\left(c_{i}^{2}(y) / y \rho_{i}, \phi-\phi_{i}\right)}{\sqrt{ }\left(\rho_{i}^{2}-c_{i}^{2}(y)\right)} \frac{d c_{i}(y)}{d y} q_{i} d S_{i} \\
& \quad=L\left(\frac{\eta}{y}\right) \frac{d}{d y} \int_{0}^{y} \frac{\rho d \rho}{\sqrt{ }\left(y^{2}-\rho^{2}\right)} L(\rho / \eta) v_{1}(\rho, \phi) .
\end{aligned}
$$

Here, the following rule of change of order of integration was used:

$$
\int_{0}^{y} d \rho \int_{0}^{c_{i}(\rho)} d x=\int_{0}^{c_{i}(y)} d x \int_{g_{i}(x)}^{y} d \rho
$$

One can easily notice that function $g_{i}(x)$ is inverse to the function $c_{i}(\rho)$. The next operator to apply is

$$
\frac{L(t / \eta)}{t} \frac{d}{d t} \int_{t}^{a_{1}} \frac{y d y}{\sqrt{ }\left(y^{2}-t^{2}\right)} L\left(\frac{\eta}{y}\right)
$$


with the result

$$
\begin{aligned}
& q_{1}(t, \phi) \\
& -\frac{1}{\pi^{2}} \sum_{i=2}^{n} \iint_{S_{i}} d S_{i}\left[\frac{L(t / \eta)}{t} \frac{d}{d t} \int_{t}^{a_{1}} \frac{y d y}{\sqrt{ }\left(y^{2}-t^{2}\right)} \frac{\lambda\left(c_{i}^{2}(y) \eta / y^{2} \rho_{i}, \phi-\phi_{i}\right)}{\sqrt{ }\left(\rho_{i}^{2}-c_{i}^{2}(y)\right)} \frac{d c_{i}(y)}{d y}\right] q_{i} \\
& =-\frac{1}{\pi^{2}} \frac{L(t / \eta)}{t} \frac{d}{d t} \int_{t}^{a_{1}} \frac{y d y}{\sqrt{ }\left(y^{2}-t^{2}\right)} L\left(\frac{\eta^{2}}{y^{2}}\right) \frac{d}{d y} \int_{0}^{y} \frac{\rho d \rho}{\sqrt{ }\left(y^{2}-\rho^{2}\right)} L(\rho / \eta) v_{1}(\rho, \phi) .
\end{aligned}
$$

Integration with respect to $y$ can be performed in (11) to give

$$
q_{1}(t, \phi)=-\frac{1}{\pi^{2}} \sum_{i=2}^{n} \iint_{S_{i}} K_{1 i}\left(t, \phi, \rho_{i}, \phi_{i}, z_{i}\right) q_{i} d S_{1}+\frac{1}{\pi^{2}} M_{1} v_{1}(t, \phi),
$$

where the kernel can be expressed in elementary functions:

$$
\begin{gathered}
K_{1 i}\left(t, \phi, \rho_{i}, \phi_{i}, z_{i}\right)=\frac{\left|z_{i}\right|}{R_{l i}^{3}}\left[\frac{R_{1 i}}{\xi_{1 i}}+\tan ^{-1} \frac{\xi_{1 i}}{R_{1 i}}\right] \\
\xi_{1 \imath}=\sqrt{ }\left(a_{1}^{2}-t^{2}\right) \sqrt{ }\left(a_{1}^{2}-c_{i}^{2}\left(a_{1}\right)\right) / a_{1} \\
R_{1 \mathrm{i}}=\left[t^{2}+\rho_{i}^{2}-2 t \rho_{i} \cos \left(\phi-\phi_{i}\right)+z_{i}^{2}\right]^{1 / 2}
\end{gathered}
$$

and

$$
\begin{aligned}
& M_{1} v_{1}(t, \phi) \\
& \quad=-\frac{L(t / \eta)}{t} \frac{d}{d t} \int_{t}^{a_{1}} \frac{y d y}{\sqrt{ }\left(y^{2}-t^{2}\right)} L\left(\frac{\eta^{2}}{y^{2}}\right) \frac{d}{d y} \int_{0}^{y} \frac{\rho d \rho}{\sqrt{ }\left(y^{2}-\rho^{2}\right)} L(\rho / \eta) v_{1}(\rho, \phi) .
\end{aligned}
$$

Similar equations can be derived for the other disks, thus forming a set of integral equations to be solved. One has to remember that each such equation is valid in the local set of coordinates related to the particular disk. It is also important to notice that during the derivation we only used the assumption that $S_{1}$ was a circular disk; (12) would remain unchanged if $S_{i}(i>1)$ were arbitrary surfaces. It is possible to prove that the set of equations (12) can be solved by successive iterations, but the most interesting feature of these equations is the ability to obtain the estimation for some integral characteristics without solving the equations.

For example, the estimation of the total charge can be made in the following manner. Multiplying both sides of (11) by $t d t d \phi$ and integrating over the surface of the first disk, one gets

$$
Q_{1}+\frac{2}{\pi} \sum_{i=2}^{n} \iint_{S_{i}} \sin ^{-1} \frac{c_{i}\left(a_{1}\right)}{\rho_{i}} q_{i} d S_{i}=\frac{1}{\pi^{2}} \int_{0}^{2 \pi} \int_{0}^{a_{1}} \frac{v_{1}(\rho, \phi) \rho d \rho d \phi}{\sqrt{ }\left(a_{1}^{2}-\rho^{2}\right)} .
$$


Introducing a new quantity $b_{i}(\rho)$ as

$$
b_{i}(\rho)=\frac{1}{2}\left\{\left[\left(\rho+\rho_{i}\right)^{2}+z_{i}^{2}\right]^{1 / 2}+\left[\left(\rho-\rho_{i}\right)^{2}+z_{i}^{2}\right]^{1 / 2}\right\},
$$

with an obvious property $c_{i}(\rho) b_{i}(\rho)=\rho \rho_{i}$, expression (14) can be rewritten in the form

$$
Q_{1}+\frac{2}{\pi} \sum_{i=2}^{n} \iint_{S_{1}} \sin ^{-1} \frac{a_{1}}{b_{i}\left(a_{1}\right)} q_{i} d S_{i}=\frac{1}{\pi^{2}} \int_{0}^{2 \pi} \int_{0}^{\hat{\mu}_{1}} \frac{v_{1}(\rho, \phi) \rho d \rho d \phi}{\sqrt{ }\left(a_{1}^{2}-\rho^{2}\right)} .
$$

Evoking the mean value theorem, which is valid when $q_{i}$ does not change sign, expression (14) can be evaluated as follows:

$$
Q_{1}+\frac{2}{\pi} \sum_{i=2}^{n} Q_{i} \sin ^{-1} \frac{a_{1}}{b_{\imath 1}}=B_{1}
$$

where $Q_{i}$ stands for the total charge of the $i$ th disk, and

$$
\begin{aligned}
& B_{1}=\frac{1}{\pi^{2}} \int_{0}^{2 \pi} \int_{0}^{a_{1}} \frac{v_{1}(\rho, \phi) \rho d \rho d \phi}{\sqrt{ }\left(a_{1}^{2}-\rho^{2}\right)} \\
& b_{i 1}=\frac{1}{2}\left\{\left[\left(a_{1}+\rho_{i 1}\right)^{2}+z_{i 1}^{2}\right]^{1 / 2}+\left[\left(a_{1}-\rho_{i 1}\right)^{2}+z_{i 1}^{2}\right]^{1 / 2}\right\} .
\end{aligned}
$$

The physical meaning of $b_{i 1}$ is obvious: it represents half of the sum of distances from a point inside $S_{i}$ to the closest and the farthermost points of the first disk's edge.

Equations similar to (17) can be derived for the other disks, and the following set of linear algebraic equations with respect to the total charges $Q_{i}$ can be written:

$$
Q_{k}+\frac{2}{\pi} \sum_{\substack{i=1 \\ i \neq k}}^{n} Q_{i} \sin ^{-1}\left(a_{k} / b_{\imath k}\right)=B_{k}, \quad k=1,2, \ldots, n,
$$

where

$$
\begin{aligned}
B_{k} & =\frac{1}{\pi^{2}} \int_{0}^{2 \pi} \int_{0}^{a_{k}} \frac{v_{k}(\rho, \phi) \rho d \rho d \phi}{\sqrt{ }\left(a_{k}^{2}-\rho^{2}\right)}, \\
b_{i k} & =\frac{1}{2}\left\{\left[\left(a_{k}+\rho_{i k}\right)^{2}+z_{i k}^{2}\right]^{1 / 2}+\left[\left(a_{k}-\rho_{i k}\right)^{2}+z_{\imath k}^{2}\right]^{1 / 2}\right\} .
\end{aligned}
$$

Of course, the exact values of $\rho_{i k}$ and $z_{i k}$ are not known, but the fact that $\left(\rho_{i k}, z_{i k}\right) \subset S_{i}$ allows us to obtain upper and lower bounds for the total charges, by solving the set (2) for the extreme points. It will be shown in the next section that in many cases this estimation is sufficiently sharp and can be used for verification of the accuracy of various approximate solutions. Notice also that in the case $v_{k}(\rho, \phi)=v_{k}=$ const.,

$$
M_{k} v_{k}(\rho, \phi)=v_{k} / \sqrt{ }\left(a_{k}^{2}-\rho^{2}\right), \quad B_{k}=\frac{2}{\pi} v_{k} a_{k} .
$$


Since $b_{k k}=a_{k}$ the set of equations (20) can be rewritten in a uniform manner

$$
\frac{2}{\pi} \sum_{i=1}^{n} Q_{i} \sin ^{-1} \frac{a_{k}}{b_{i k}}=B_{k}, \quad k=1,2, \ldots, n .
$$

The possibility of assessing the integral characteristics in such a simple manner is not limited to the quantity of total charge. One can multiply (11) by $t^{m} d t d \phi$ and integrate over the surface $S_{1}$. The result can always be expressed in terms of elementary functions. For example, in the case $m=2$, the result of integration is

$$
\begin{aligned}
& \int_{0}^{2 \pi} \int_{0}^{a_{1}} q_{1}(t, \phi) t^{2} d t d \phi+\sum_{i=2}^{n} \iint_{S_{i}}\left[\sqrt{ }\left(\rho_{i}^{2}+z_{i}^{2}\right)\right. \\
& \left.-\sqrt{ }\left(b_{i}^{2}\left(a_{1}\right)-a_{1}^{2}\right)+\left|z_{i}\right| \ln \frac{b_{i}\left(a_{1}\right)\left[\sqrt{ }\left(b_{i}^{2}\left(a_{1}\right)-a_{1}^{2}\right)+\left|z_{i}\right|\right]}{\left.\sqrt{ }\left(b_{i}^{2}\left(a_{1}\right)-a_{1}^{2}\right)|| z_{i} \mid+\sqrt{ }\left(\rho_{i}^{2}+z_{i}^{2}\right)\right]}\right] q_{i} d S_{i} \\
& \quad=\frac{1}{2 \pi} \int_{0}^{2 \pi} \int_{0}^{a_{1}}\left[\frac{a_{1}}{\sqrt{ }\left(a_{1}^{2}-\rho^{2}\right)}-\cosh ^{-1} \frac{a_{1}}{\rho}\right] v_{1}(\rho, \phi) \rho d \rho d \phi .
\end{aligned}
$$

Here, one can again evoke the mean value theorem and get upper and lower bounds for the quantities of interest.

\section{Examples}

EXAMPLE 1. The simplest example to consider is the case of two disks of radii $R_{1}$ and $R_{2}$, lying in two planes intersecting at an angle $\alpha$, and whose centres are lying in one plane orthogonal to the line of intersection at the distances $l_{1}$ and $l_{2}$ from the line. Let the disks be conductors charged to the potentials $V_{1}$ and $V_{2}$ respectively. The total charges $Q_{1}$ and $Q_{2}$ are to be determined. The set of equations to be solved has the form

$$
\begin{aligned}
& Q_{1}+\frac{2}{\pi} Q_{2} \sin ^{-1} \frac{a_{1}}{b_{21}}=\frac{2}{\pi} V_{1} a_{1}, \\
& \frac{2}{\pi} Q_{1} \sin ^{-1} \frac{a_{2}}{b_{12}}+Q_{2}=\frac{2}{\pi} V_{2} a_{2},
\end{aligned}
$$

where

$$
\begin{gathered}
b_{21}=\frac{1}{2}\left\{\left[\left(l_{2}+x\right)^{2}+\left(l_{1}-R_{1}\right)^{2}-2\left(l_{2}+x\right)\left(l_{1}-R_{1}\right) \cos \alpha\right]^{1 / 2}\right. \\
\left.+\left[\left(l_{2}+x\right)^{2}+\left(l_{1}+R_{1}\right)^{2}-2\left(l_{2}+x\right)\left(l_{1}+R_{1}\right) \cos \alpha\right]^{1 / 2}\right\} \\
b_{12}=\frac{1}{2}\left\{\left[\left(l_{1}+y\right)^{2}+\left(l_{2}-R_{2}\right)^{2}-2\left(l_{1}+y\right)\left(l_{2}-R_{2}\right) \cos \alpha\right]^{1 / 2}\right. \\
\left.+\left[\left(l_{1}+y\right)^{2}+\left(l_{2}+R_{2}\right)^{2}-2\left(l_{1}+y\right)\left(l_{2}+R_{2}\right) \cos \alpha\right]^{1 / 2}\right\}
\end{gathered}
$$


with $-R_{2} \leq x \leq R_{2}$, and $-R_{1} \leq y \leq R_{1}$. The extrcme points give the upper and the lower bounds for the total charges. It is logical to consider the central estimation corresponding to $x=y=0$. Calculations show that in some cases the central estimation is very close to the exact result.

The problem of two non-parallel disks was considered in [3] using the MehlerFok transform. The following result was obtained for the total charge $Q_{1}$ on the assumption that

$$
\begin{gathered}
\mu_{1} / \sin \frac{\alpha}{2} \ll 1 \quad \text { and } \mu_{2} / \sin \frac{\alpha}{2} \ll 1 ; \quad \mu_{1}=R_{1} / l_{1}, \quad \mu_{2}=R_{2} / l_{2} \\
Q_{1} \approx \frac{2}{\pi} R_{1}\left[V_{1}-V_{2} \frac{\mu_{2}}{\pi \sin \alpha / 2}+V_{1} \frac{\mu_{1} \mu_{2}}{\pi^{2} \sin ^{2} \alpha / 2}-V_{2} \frac{\mu_{1} \mu_{2}^{2}}{\pi^{3} \sin ^{3} \alpha / 2}\right. \\
\left.+V_{2} \frac{\mu_{1}^{2} \mu_{2}\left(2+3 \sin ^{2} \alpha / 2\right)}{24 \pi \sin ^{3} \alpha / 2}+V_{2} \frac{\mu_{2}^{3}\left(2-9 \sin ^{2} \alpha / 2\right)}{24 \pi \sin ^{3} \alpha / 2}\right] .
\end{gathered}
$$

It is of interest to compare the results given by $(25-26)$ with those by Rukhovets and Uflyand (27). Calculations were performed for the case $R_{1}=R_{2}=1$, $V_{1}=V_{2}=1, \alpha=\pi / 4, l_{1}=l_{2}=l$. The value of $Q_{1}^{*}=\pi Q_{1} / 2$ versus $l$ is presented in Figure 1. The upper and the lower bounds are given by the solid and the dashed lines respectively. The solid circle line gives the central estimation; the small circle line represents the results of evaluating (27). As one can see, (27) gives good results for $l>2.5$; the results sharply deviate from the admissible zone for $l<2$. Our term 'admissible zone' indicates the interval between the upper and the lower bounds. The central estimation gives reasonably good accuracy in the whole range $0<l<\infty$, except the narrow interval near $l=1$ where the worst possible error is about $25 \%$.

The real value of the error can be found only by comparison with the exact result, which is not available at the moment, but there is a reason to believe that the error is much smaller than the value quoted above. This belief is based on the only numerical computations known to us, which claim to be accurate, namely those due to Kobayashi [2], who solved the problem of two equal coplanar disks kept at constant unit potentials. The case of coplanar disks is covered by (25-26) taking $\alpha=\pi$. The comparison of our results with those by Kobayashi is given in Table 1. The central estimation error was evaluated with respect to the Kobayashi result which was assumed to be exact. The error value is surprisingly small, but the fact that the error sign changes indicates that Kobayashi's results are not exact. Indeed, it may be proved that the exact value of $b_{21}$ is greater than $2 l$, due to the charge interaction (repulsion in this case); therefore, the central estimation in (25) is, in fact, a very close upper bound. This means that the error sign should be negative, and changing monotonically (decreasing) as the distance between the disks increases. The only definite conclusion which can be drawn from this comparison is that our very simple approach gives the same 


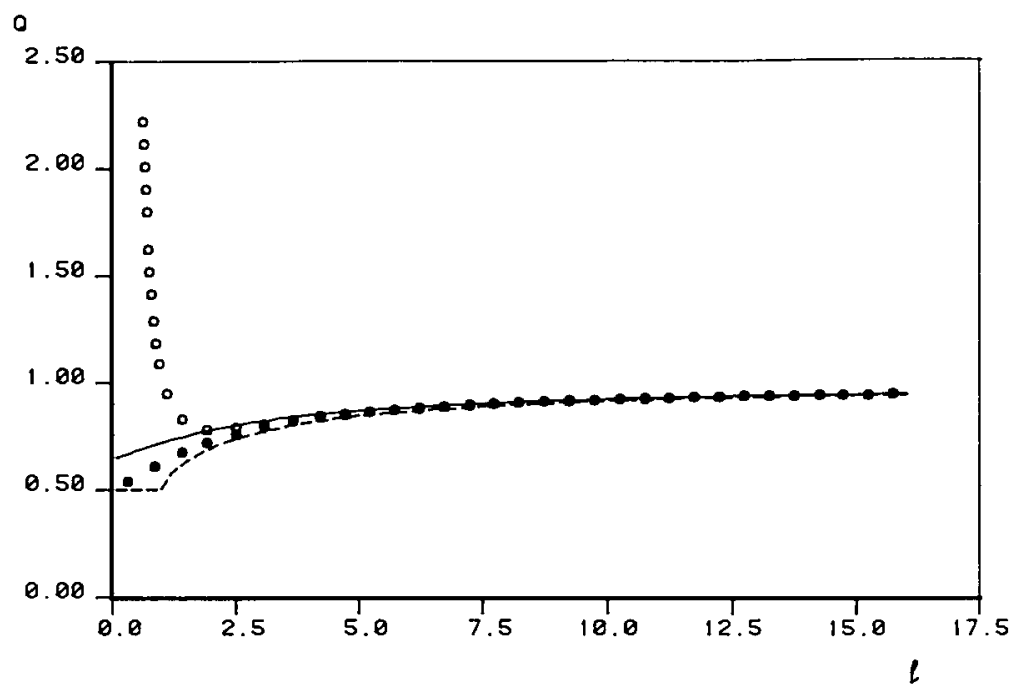

Figure 1. Two nonparallel disks at unit potential

accuracy as the method using the double series expansions, infinite systems of linear algebraic equations whose coefficients are given by complicated integrals involving special functions.

TABLE 1. Evaluation of the total charge (two equal coplanar disks)

\begin{tabular}{cllllc}
\hline $\begin{array}{l}\text { Distance } \\
\text { Between } \\
\text { Centres }\end{array}$ & $\begin{array}{l}\text { Upper Bound } \\
\text { for the Total } \\
\text { Charge }\end{array}$ & $\begin{array}{l}\text { Lower Bound } \\
\text { for the Total } \\
\text { Charge }\end{array}$ & $\begin{array}{l}\text { Central } \\
\text { Estimate }\end{array}$ & $\begin{array}{l}\text { Kobayashi's } \\
\text { Results }\end{array}$ & $\begin{array}{l}\text { Error of the } \\
\text { Central } \\
\text { Estimate } \\
\%\end{array}$ \\
\hline 2.0 & 0.8221338 & 0.5000000 & 0.7500000 & 0.75272 & .36 \\
2.2 & 0.8317164 & 0.6145749 & 0.7689962 & 0.77014 & .15 \\
2.4 & 0.8402998 & 0.6637918 & 0.7851738 & 0.78545 & .04 \\
2.6 & 0.8480356 & 0.6993975 & 0.7991486 & 0.79898 & -.02 \\
2.8 & 0.8550458 & 0.7272786 & 0.8113602 & 0.81096 & -.05 \\
3.0 & 0.8614294 & 0.7500000 & 0.8221338 & 0.82162 & -.06 \\
3.5 & 0.8751494 & 0.7924057 & 0.8442654 & 0.84370 & -.07 \\
4.0 & 0.8863767 & 0.8221338 & 0.8614294 & 0.86093 & -.06 \\
5.0 & 0.9036683 & 0.8614294 & 0.8863767 & 0.88602 & -.04 \\
7.0 & 0.9261093 & 0.9036683 & 0.9163737 & 0.91619 & -.02 \\
10.0 & 0.9452202 & 0.9338098 & 0.9400541 & 0.9399 & -.007 \\
$\infty$ & 1. & 1. & 1. & 1. & 0 \\
\hline
\end{tabular}

EXAMPLE 2. Consider the case of $n+1$ disks with their centres located at the plane $z=0$. The plane of the first disk is horizontal, its centre being placed at the coordinate system origin, and its radius being $a_{0}$. This disk will be called central. The centres of the remaining $n$ equal disks are located at the vertices of a regular polygon, their planes being orthogonal to the line connecting the 
coordinate's origin with the vertex, the length of this line being $l$. Let the central disk be charged to a potential $V_{0}$, the rest being kept at a potential $V_{1}$, and their radii being $a_{1}$. Due to the symmetry of the system, the problem of finding the total charge at each disk can be reduced to a set of just two linear algebraic equations:

$$
\begin{gathered}
Q_{0}+\frac{2}{\pi} n Q_{1} \sin ^{-1} a_{0} / b_{0}=\frac{2}{\pi} V_{0} a_{0}, \\
\frac{2}{\pi} Q_{0} \sin ^{-1} a_{1} / b_{01}+Q_{1}\left[1+\frac{2}{\pi} \sum_{i=2}^{n} \sin ^{-1} a_{1} / b_{i 1}\right]=\frac{2}{\pi} V_{1} a_{1},
\end{gathered}
$$

where, from elementary geometrical considerations,

$$
\begin{aligned}
b_{0} & =\frac{1}{2}\left\{\left[\left(l+a_{0}\right)^{2}+x^{2}\right]^{1 / 2}+\left[\left(l-a_{0}\right)^{2}+x^{2}\right]^{1 / 2}\right\} \\
b_{01} & =\left[(l-y)^{2}+a_{1}^{2}\right]^{1 / 2}, \\
b_{i 1} & =\frac{1}{2}\left[\sqrt{ }\left(x_{i 1}^{2}+y_{i 1}^{2}\right)+\sqrt{ }\left(X_{i 1}^{2}+Y_{i 1}^{2}\right)\right] \\
x_{i 1} & =2 h \sum_{k=1}^{i-2} \sin 2 \pi k / n+\left(h-a_{1}\right) \sin \frac{2 \pi(i-1)}{n}, \quad h=l \tan \pi / n \\
y_{i 1} & =2 h \sum_{k=1}^{i-2} \cos 2 \pi k / n+\left(h-a_{1}\right) \cos 2 \pi(i-1) / n+(h+x), \\
X_{i 1} & =2 h \sum_{k=1}^{i-2} \sin 2 \pi k / n+\left(h+a_{1}\right) \sin 2 \pi(i-1) / n, \\
Y_{i 1} & =2 h \sum_{k=1}^{i-2} \cos 2 \pi k / n+\left(h+a_{1}\right) \cos 2 \pi(i-1) / n+(h+x),
\end{aligned}
$$

with $-a_{1}<x<a_{1}$ and $-a_{0}<y<a_{0}$. The central estimation corresponds to the case $x=y=0$. Note that the accuracy of the central estimation improves, as the ratios $l / a_{0}$ and $l / a_{1}$ increase, tending to the exact results when these ratios tend to infinity.

As one can see, the new approach allows a very simple treatment of complicated problems. The method is not limited to circular disks; it can be modified for other surfaces. For example, a system of arbitrarily located spherical caps can be treated in a similar manner. 


\section{References}

[1] V. I. Fabrikant, "A new approach to some problems in potential theory", Z. Angew. Math. Mech. 66 (1986) 363-368.

[2] L. Kobayashi, "Das electrostatische Potential um zwei auf derselben Eben liegende und sich nicht schneidende gleichgroße Kreisscheiben," Sci. Rep. Tôhoku Imp. Univ. 27 (1939) 365-391.

[3] A. N. Rukhovets and Ya. S. Uflyand, "Electrostatic field of a system of non-parallel circular disks", Soviet Physics. - Technical Physics, 41, No. 6 (1971) 1103-1108.

[4] I. N. Sneddon, Mixed Boundary Value Problems in Potential Theory (Amsterdam, 1966).

[5] Ya. S. Uflyand, Method of Coupled Equations in Mathematical Physics Problems (in Russian) (Academy of Sciences of the USSR, Nauka, Leningrad, 1977). 